\title{
Flowrate measurements of conducting fluids in pipes using the magnetic distortion probe
}

\author{
Guillaume Bousselin, Marius Tanase, Marc Moulin and Nicolas \\ Plihon
}

Univ Lyon, ENS de Lyon, Univ Claude Bernard Lyon 1, CNRS, Laboratoire de

Physique, F-69342 Lyon, France

E-mail: nicolas.plihon@ens-lyon.fr

May 2017

\begin{abstract}
A contactless and local velocimetry technique based on the interpretation of the induced magnetic field in the presence of a strongly localised applied magnetic field in the vicinity of flowing electrically conducting fluids, refereed to as the magnetic distortion probe, has been introduced in Miralles et al., Rev. Sci. Instrum., 82095112 (2011). In this article, we present a numerical model, shown to accurately predict the magnetic distortion probe response measured in a dedicated experimental pipe flow benchmark. This numerical model allows to precisely estimate the calibration of the magnetic distortion probe. This model is also used to investigate the spatial extent of the flow probed by the magnetic distortion probe. The optimal arrangement required to probe global flowrates in pipes is finally discussed based on the numerical model.
\end{abstract}

Submitted to: Meas. Sci. Technol.

\section{Introduction}

Velocity measurements in electrically conducting liquids is an important issue for control and characterisation of flows in various situations, ranging from the control of liquid metals used as coolant in fast breeder fission reactor to processes in the chemical and food-processing industries [1]. However, this is usually a challenging task because of the opacity, high chemical reactivity and usually high ambient temperature of the liquid metals or chemical mixes [2,3]. Non invasive techniques developed for neutral fluids, such as ultrasonic methods [4, 5] or neutron radiography [6] have been adapted for liquid metals. These techniques are indirect flow measurements techniques since they rely on the scattering of waves or particles by tracer impurities or bubbles and have major drawbacks such as difficult implementation or costly operation. Several methods relying on the interpretation of the interaction between a moving electrically conducting fluid with externally applied magnetic fields have also been proposed. An eddy-current flow meter, proposed as early as 1948 [7] is based on the measurement 
of the advection of a magnetic field, created by two coils fed by an AC current. This technique is still currently in use [8]. Magnetic phase shifts introduced by the flow in similar configurations may also be used to probe velocities or flow rates [9]. A global tomographic method was demonstrated to reconstruct $2 \mathrm{D}$ or $3 \mathrm{D}$ features of the flows from the interpretation of induced magnetic field distributions from the velocity field distribution [10, 11]. More recently, two techniques relying on the interaction of magnetic fields with flowing conducting fluids have been introduced. The first one interprets the force acting on a permanent magnet in the vicinity of an electrically conducting fluid flow, due to the interaction of the induced magnetic field - created by currents induced in the flow - with the magnetic moment of the permanent magnet. Several configurations have been tested: the Lorentz Force Velocimetry technique probes flow rates in pipes [12], while the Local Lorentz Flow Velocimetry probes the velocity at one point thanks to the use of a miniature permanent magnet [13]. On the other hand the magnetic distortion probe $[14,15]$, probes the induced magnetic field in the vicinity of the permanent magnet. The latter technique allows to achieve very good temporal response and to include corrections from external spurious magnetic fields.

The magnetic distortion probe is based on the interpretation of the induced magnetic field $\mathbf{B}^{\mathbf{i}}$ in the vicinity of a localised externally applied magnetic field $\mathbf{B}^{\mathbf{0}}$ and an electrically conducting fluid flow at velocity $\mathbf{u}$. This technique was initially developed to measure local fluid velocities (with a 2D resolution in Ref. [14]) implementing a permanent magnet as the localised applied magnetic field. In this article, we investigate its ability to measure flowrates in pipes. Using an Hallbach array of permanent magnet, experimental measurements of flow rates in an benchmark pipe flow are reported in section 2. A numerical model is introduced in section 2, and very good agreement between the experimental data and the simulations are observed. We show that this numerical model may be used to compute a calibration coefficient taking into account the exact geometry of the probe and of the pipe flow. This model may also be used to optimise the probe design in order to probe flowrates, as demonstrated in section 3. Finally, the issue of the spatial resolution of measurements made with the magnetic distortion probe in the configuration used in Ref. [14] is addressed in section 4. Concluding remarks are provided in section 5

\section{Measurements in a liquid gallium pipe flow}

\subsection{Experimental setup}

A mid-size gallium pipe flow is created between two tanks connected by a $1 \mathrm{~m}$ long, $D=10 \mathrm{~mm}$ inner diameter and $1 \mathrm{~mm}$ thick amagnetic stainless steel pipe. The upper tank may be pressurised at pressure $P$ up to 3 bar with Argon and an electrically controlled valve allow liquid gallium, of electrical conductivity $\sigma=3.810^{6} \mathrm{~S}^{-\mathrm{m}^{-1}}$, density $\rho=6.095$ g.cm ${ }^{-3}$ and temperature around $50^{\circ} \mathrm{C}$, to flow within the pipe to the lower tank. The velocity of the flow follows Torricelli's law and strain gauges placed 
on the upper tank allow the measurement of the mass $m(t)$ of gallium exiting the tank. The loop contains $8 \mathrm{~L}$ of liquid gallium and steady regimes from 5 to $25 \mathrm{~s}$ are achieved depending on the value of $P$. Following the magnetic distortion probe sensing method proposed by Miralles et coworkers [14], a set of permanent magnet is placed in the vicinity of the pipe, creating an externally applied magnetic field $\mathbf{B}^{\mathbf{0}}$. A commercial Hall sensor (GW Bell 7030) probes the value of the induced magnetic field $\mathbf{B}^{\mathbf{i}}$ in the direction of the flow. A sketch of the experimental setup is provided in Fig. 1 and shows an Hallbach type array implementing ten NdFeB magnets $(10 \mathrm{~mm} \times 10 \mathrm{~mm} \times 40 \mathrm{~mm}$, magnetised along one of the smallest direction) placed $7.5 \mathrm{~mm}$ away from the outer edge of pipe. The motivation to choose an Hallbach array [16] relies on (i) the fact that the amplitude of the magnetic distortion probe signal is proportional to the gradient of the applied magnetic field in the flow direction (Hallbach arrays allow to impose very strong gradients), (ii) the high spanwise homogeneity in the applied magnetic field, (iii) the very low value of applied magnetic field outside the array. Note that similar arrangements have been proposed to probe flowrates of very low electrical conductivity electrolytes using Lorentz force velocimetry [17]. The Hall sensor probes the induced magnetic field component $B_{x}^{i}$ in the flow direction at $3 \mathrm{~mm}$ from the pipe and can be moved along the $x$ axis from $x=-50$ to $x=50 \mathrm{~mm}$ (the origin of the $x$-axis lying in the center of the Hallbach array). Signals are sampled at a rate of $1000 \mathrm{~Hz}$, with 16 bits resolution.

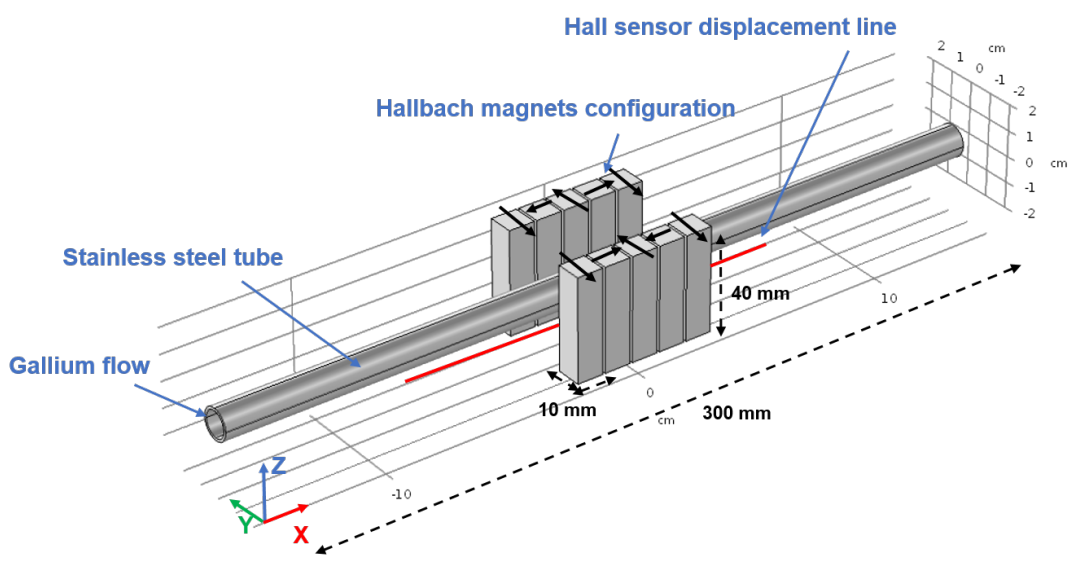

Figure 1. Sketch of the experimental arrangement of the magnetic distortion probe using an Hallbach array in the vicinity of a gallium pipe flow. Liquid gallium is flowing along the $x$ direction. Arrows indicate the magnetisation direction of each of the magnets of the Hallbach array. 


\subsection{Numerical model}

The finite element solver Comsol Multiphysics has been used to compute the magnetic field induced by the coupling between the liquid metal flow and an external magnetic field. The coupled set of Navier Stokes and Maxwell equations are solved using Comsol's Computational Fluid Dynamcis (CFD) and electromagnetic AC/DC modules. This technique was shown to accurately predict or reproduce features of MHD flows at low magnetic Reynolds numbers [18, 19] and high magnetic Reynolds number [20].

The geometry implemented in the numerical simulations is identical to that of the experimental setup. Liquid gallium with electrical conductivity $\sigma$ and density $\rho$ is flowing inside a $10 \mathrm{~mm}$ inner diameter, $1 \mathrm{~mm}$ thick and $300 \mathrm{~mm}$ long stainless steel pipe. The externally applied magnetic field is generated by the Hallbach configuration introduced earlier (see Fig. 1). Each permanent magnet is a rectangular parallelepiped of dimension $10 \times 10 \times 40 \mathrm{~mm}$, having a remanent flux density $B_{r}=1.310^{4} \mathrm{G}$ according to the NdFeB N42 magnet grade used in the experimental arrangement, and is magnetised along one of the smallest direction (see Fig. 1), alternatively along the $y$ and $x$ directions. The Comsol CFD module is used to solve the Navier Stokes equation in the fluid domain for an incompressible and stationary gallium flow:

$$
\begin{aligned}
& \nabla \cdot \mathbf{u}=0 \\
& \rho(\mathbf{u} . \nabla) \mathbf{u}=\nabla \cdot\left[-p \mathbf{I}+\mu\left(\nabla \mathbf{u}+(\nabla \mathbf{u})^{T}\right)\right]+\mathbf{F}
\end{aligned}
$$

with $\mathbf{u}$ the liquid gallium velocity field, $\mu=1.810^{-3}$ Pa.s the dynamic viscosity, $p$ the pressure and $\mathbf{F}$ the Lorentz force. The flowrate is imposed at the tube inlet and null pressure at the outlet.

The velocity field is then supplied to the AC/DC module which computes the induced current according to the generalised Ohm's law $\mathbf{J}=\sigma(-\nabla V+\mathbf{u} \times \mathbf{B})$ in the different domains of the model; $V$ being the electric potential and $\mathbf{B}=\mu_{0} \mathbf{H}+B_{r} \mathbf{b}$ where $\mathbf{b}$ is the magnetisation direction of each magnet (we recall that the remanent flux density $B_{r}$ is non-zero in the magnets domain only). This is solved imposing Ampere's law and the current conservation equation:

$$
\nabla \times \mathbf{H}=\mathbf{J} \quad ; \quad \nabla . \mathbf{J}=0
$$

Continuity conditions are used for the internal boundaries between internal domains refereed to as $\alpha$ and $\beta$ :

$$
\mathbf{n} \times\left(\mathbf{H}_{\alpha}-\mathbf{H}_{\beta}\right)=0 \quad ; \quad \mathbf{n} \times\left(\mathbf{J}_{\alpha}-\mathbf{J}_{\beta}\right)=0
$$

and magnetic insulation conditions are specified for the external boundaries of the model:

$$
\mathbf{n} \times \mathbf{A}=0 ; \quad V=0
$$

where $\mathbf{A}$ is the magnetic vector potential. The induced magnetic field is then computed from the flow-induced currents $\mathbf{J}$. 
The coupling between the $\mathrm{CFD}$ and the $\mathrm{AC} / \mathrm{DC}$ modules is repeated until convergence is found. At first, the CFD module is run discarding the Lorentz force (i.e. $\mathbf{F}=\mathbf{0}$ ) to solve for a purely hydrodynamic velocity profile. This purely hydrodynamic velocity field is then supplied to the $\mathrm{AC} / \mathrm{DC}$ module which computes the induced current. The Lorentz force, computed from the interaction of the induced current with the applied magnetic field, is then fed into the CFD module for the subsequent computations.

The entire model has been meshed using free tetrahedral elements with a heterogeneous density. The mesh density has been increased in the fluid domain as well as in the air domain between the magnets assembly and the pipe edge to account for the strong gradients in both the velocity and magnetic fields and to increase the resolution in the region where the experimental measurements have been done. The size of the mesh elements has been chosen in order to obtain a good spatial resolution while keeping the computational time reasonable. Finally, the number of degrees of freedom is approximatively 2.6 millions.

\subsection{Experimental validation of the numerical model}

The profiles of the components of the applied magnetic field in the spanwise direction $B_{y}^{0}$ and in the streamwise direction $B_{x}^{0}$ are displayed in Fig. 2(a) and (b) respectively, for $z=0 \mathrm{~mm}$, i.e. at the altitude of the center of the pipe. The leftmost panel shows very large values of the streamwise gradient of the spanwise component of the magnetic field, of the order of $410^{5} \mathrm{Gm}^{-1}$. The very good agreements between the experimental values and numerical model, respectively displayed as blue circles and full red lines in Fig. 2, both for the components $B_{x}^{0}$ and $B_{y}^{0}$, validates the numerical model. While the $y$ (spanwise) component has even parity, the $x$ (streamwise) component has odd parity. The (red) full line curve of Fig. 2(c) displays the values of the streamwise component of the induced magnetic field $B_{x}^{i}$, which is three order lower in magnitude than the applied magnetic field. Typical time series of the streamwise component of the induced magnetic field as the valve is opened are reported in Fig. 3(a) for different probing locations along the pipe. The three reported time traces have been acquired in successive shots and show that steady-state flows are obtained typically one second after triggering the valve opening. In the remaining of this section, one will report evolution of time-averaged values of the induced magnetic field $\left\langle B_{x}^{i}\right\rangle$ in the stationary regime. As expected from Fig. 2(c), the values of the induced magnetic strongly depend on the probing location. We also note that the $x$-component of the induced magnetic field has even parity. As a consequence, the measurements of the induced magnetic field at locations where $B_{x}^{0} \sim 0$ are relatively easy. At locations where $\left|B_{x}^{0}\right|$ is maximal, very small $B_{x}^{i}$ values are induced, and accurate measurements require an extremely large resolution of the magnetic sensor (of the order of $10^{-5}$ ). The very good agreement between the experimental values and the numerical simulations of the $x$-component of the induced magnetic field $B_{x}^{i}$ reported in Fig. 2(c) validates the accuracy of the numerical model coupling the resolution of the 
flow dynamics and electromagnetism.
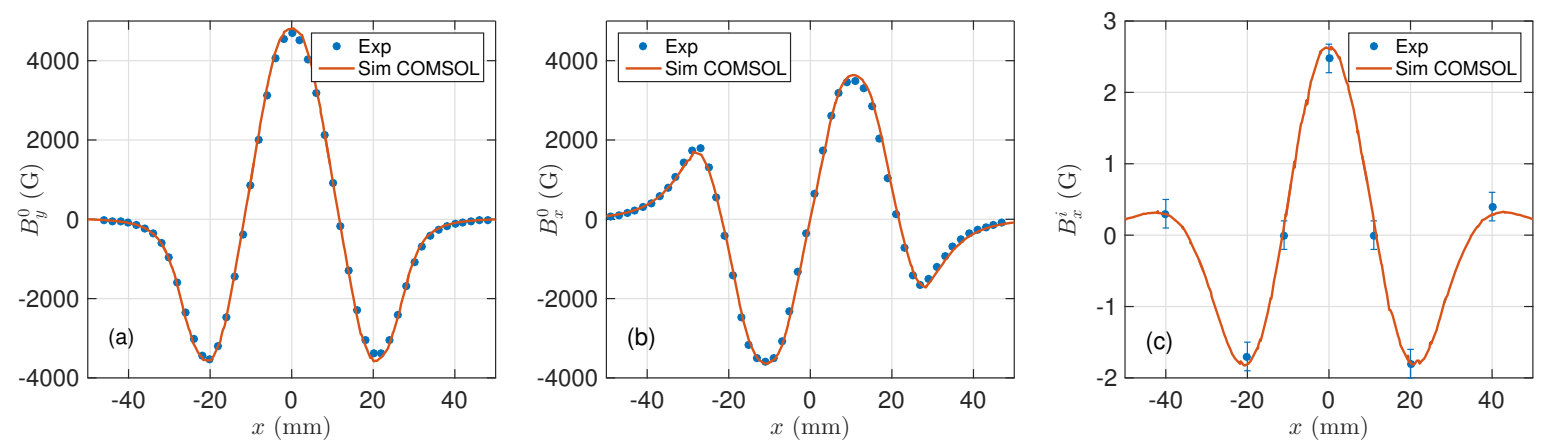

Figure 2. Profiles of experimental values and numerical simulations of (a) the $y$ component (spanwise) imposed magnetic field $B_{y}^{0}$, (b) the $x$-component (streamwise) imposed magnetic field $B_{x}^{0}$ field and (c) the $x$-component (streamwise) induced magnetic field $B_{x}^{i}$ along the $x$ axis for a $Q=0.2 \mathrm{~L} / \mathrm{s}$ flowrate.

Let us now investigate how the maximum value of the time-averaged induced magnetic field $\left\langle B_{x}^{i}\right\rangle$ (i.e. at $x=0$ ) evolves as a function of the pipe flowrate $Q$. The flowrate $Q$ is controlled by the pressure in the upper Argon tank, ranging from 0.2 to 3 bars. The flowrate $Q$ is computed from the time evolution of the upper tank gallium mass $m(t)$ as $Q(t)=d m(t) / d t$. As expected from the linearity of the induction equation, $\left\langle B_{x}^{i}\right\rangle$ scales linearly with $Q$, as shown in Fig. 3(b). Once again, a very good agreement is obtained between the experimental values and our numerical model (where $Q$ is the CFD boundary condition at the pipe inlet). It is important to note here that, in the geometry relative to the numerical simulations, the effect of the Lorentz force is small.

Due to the moderate values of the interaction parameter $N=\frac{\sigma\left(B^{0}\right)^{2} D}{2 \rho U} \sim 0.1$, the modifications of the flow profiles and of the induced magnetic field are of the order of $1 \%$ (not shown). This is still verified though the Hartmann number $H a=B^{0} D / 2 \sqrt{\sigma / \mu}$ is of the order of 50 since the length over which a strong magnetic field is imposed is small (of the order of the tube diameter $D$ ). As a partial conclusion, we showed the accuracy of our numerical model to predict the behaviour of the magnetic distortion experimentally observed in a gallium pipe flow.

\section{Spatial resolution of the magnetic distortion probe using a Hallbach array}

In order to characterise finely the ability of the magnetic distortion probe to measure flowrates of conducting fluids in pipes, the spatial resolution of the measurement is addressed using our numerical model. The magnetic distortion probe was demonstrated to be a local velocity sensor [14], and, since the velocity profile in the pipe does not evolve with velocity (nor the pipe section), the measurements presented above could 

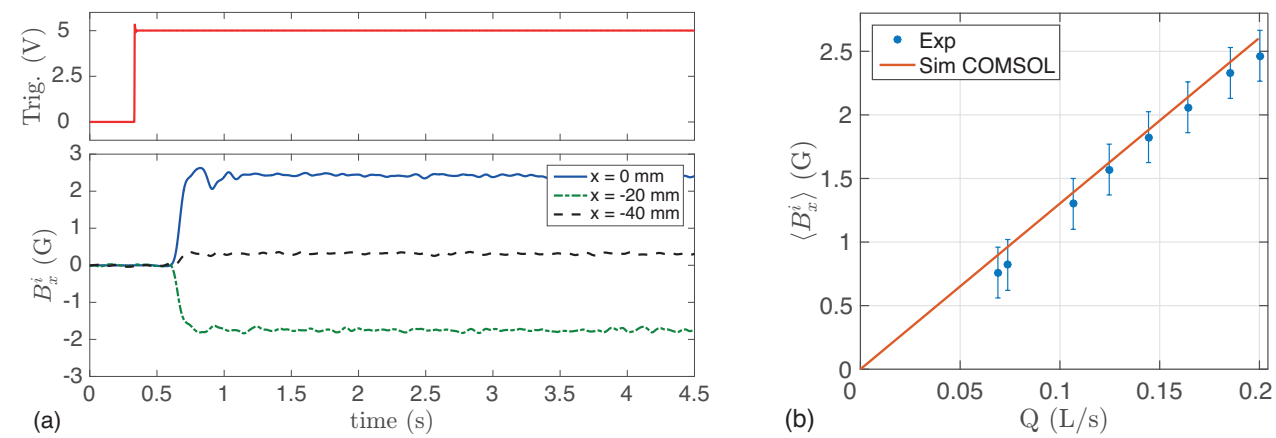

Figure 3. (a) Typical times series of the streamwise component of the induced magnetic field for three probing locations along the pipe (10 Hz low-pass filtered), obtained for the flowrate $Q=0.2 \mathrm{~L} / \mathrm{s}$. (b) Evolution of $\left\langle B_{x}^{i}\right\rangle$, at $x=0$, as a function of the pipe flowrate $Q$.

be expressed equivalently as a function of the velocity or as a function of the flowrate $Q$. The use of the magnetic distortion probe as a flowmeter thus requires a uniform contribution to the induced magnetic field from each location in the pipe section. To address this issue, a slightly modified geometry, displayed in Fig. 4, with a pipe having a square cross-section of length $10 \mathrm{~mm}$, has been used. The pipe has been divided into 36 equal cells (2 by $2 \mathrm{~mm}$ squares) and a set of numerical simulations was run where a uniform (solib-body like) flow is imposed successively in only one of the cells, the other cells being kept at rest. The magnetic configuration is that created by the Hallbach array presented in section 2. The electrical conductivity of all cells was kept constant and equal to the value of liquid gallium. The intensity of the induced magnetic field probed at location 1 when the liquid metal is flowing in only one of the the cells is displayed at that specific cell location in Fig. 4(b). These values are normalised to the highest value of all cells. It is important to note that the simulations have been led in three dimensions, but that the representation of Fig. 4(b) is in two dimensions. This is a representation of the spatial response function of the magnetic distortion probe in the spanwise direction, which shows that the configuration with one sensor at location 1 averages roughly one third of the pipe. A straightforward upgrade relies on the use of two sensors (at location 1 and location 2). The intensity of the sum of the normalised induced magnetic field is displayed in Fig. 4(c) for each of the cells. A much more homogeneous response function is obtained in this configuration. This nearly uniform response function may be used to probe the global flow rate even in the presence of flow inhomogeneities in the pipe. It is important to note that, in principle, this configuration also allow for the determination of flowrates in a pipe subject to material deposition if the electrical conductivity of the deposited material is the same as that of the fluid. In practice, this might not be verified since, for instance, deposited oxides would have a lower conductivity than the fluid. This would modify the details of how the induced currents loop back in the system, requiring 
a dedicated numerical study to determine how the accuracy of the flowrate measurement depends on the electrical conductivity and on the thickness of the deposited material. Note that more advanced and adapted configurations than the Hallbach array coupled to two magnetic sensors could be designed (using for instance a larger number of probes and optimised applied magnetic field configurations).
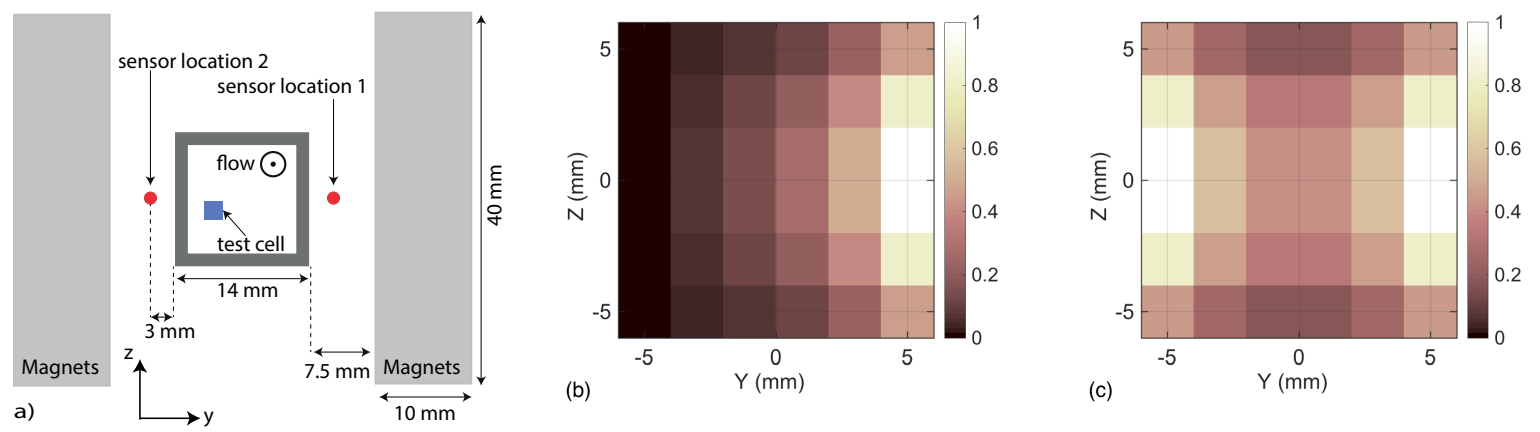

Figure 4. (a) Sketch of the geometry used to compute the spatial resolution using an Hallbach array around a square duct, see text for details. Normalised spatial resolution with (b) a single probe at location 1, (c) two sensors at locations 1 and 2.

\section{Spatial resolution of the magnetic distortion probe using a single magnet}

The proof of concept of velocity measurements in electrically conducting fluids using a single permanent magnet and magnetic field sensors probing the induced magnetic field component in the flow direction, in the vicinity of the magnet, was demonstrated in Ref. [14] in various operational configurations. In this previous work, the spatial resolution of the measurement was not investigated in details. We now provide a set of numerical simulations addressing the issue of the spanwise spatial resolution of the magnetic distortion measurement, following the method introduced in the previous section, where the flow is decomposed into elementary cells to compute the response function. A configuration with one single magnet and one magnetic field sensor, sketched in Fig. 5 (leftmost panel), has been implemented with 98 cells (leading to 49 computations due to symmetry arguments) in a rectangular pipe with $7 \times 14 \mathrm{~mm}$ inner dimensions and $1 \mathrm{~mm}$ thick stainless steel walls. The location of the magnetic sensor is set at $1 \mathrm{~mm}$ from the outer wall of the pipe, at $z=0$. A small cylindrical permanent magnet of diameter $2 \mathrm{~mm}$ and length $2 \mathrm{~mm}$ is set at a distance $D_{m}$ from the wall. The panels of Fig. 5 show the normalised spatial response, in the spanwise directions, as $D_{m}$ increases from 1 to $5 \mathrm{~mm}$ (left to right). As $D_{m}$ increases, the volume probed by the sensor increases. This can be easily understood since the applied magnetic magnetic field is much more homogeneous in the pipe for larger values of $D_{m}$ (as the applied magnetic field scales as $l^{-3}$ at a distance $l$ from the center of the magnet). Fig. 6(a) displays the evolution of the $z$-component of the induced current $J_{i}^{z}$ at $z=0$ in each cell as a function of the distance $l$ of the cell relative to the center of the magnet. 

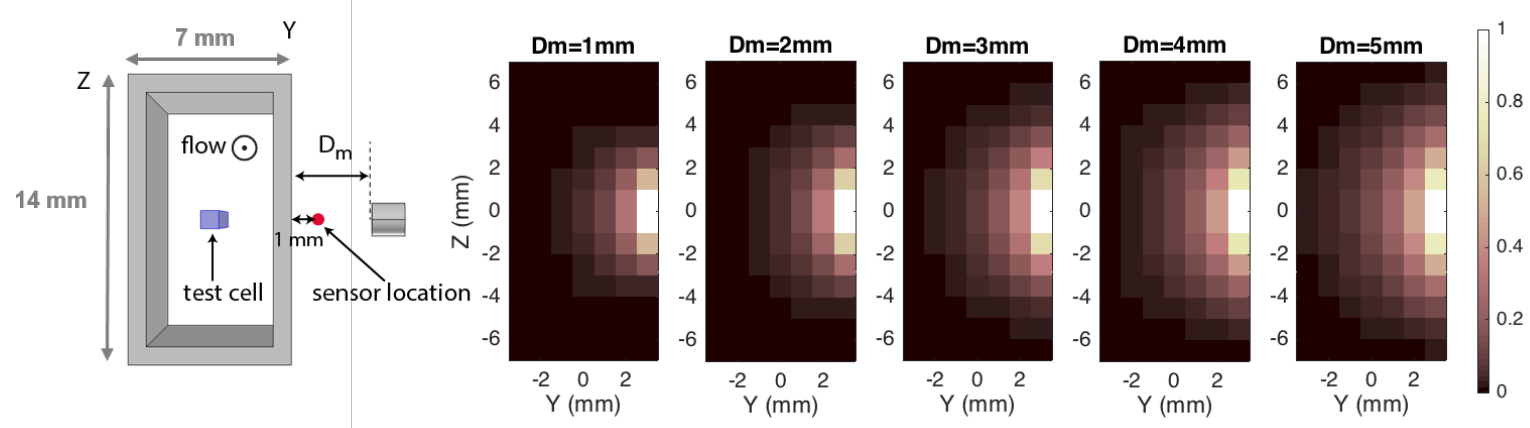

Figure 5. Leftmost panel: sketch of the geometry used to compute the spatial resolution using a single cylindrical permanent magnet facing a rectangular duct, see text for details. Normalised spatial resolution in the spanwise directions as the distance $D_{m}$ between the magnet and the pipe increases.
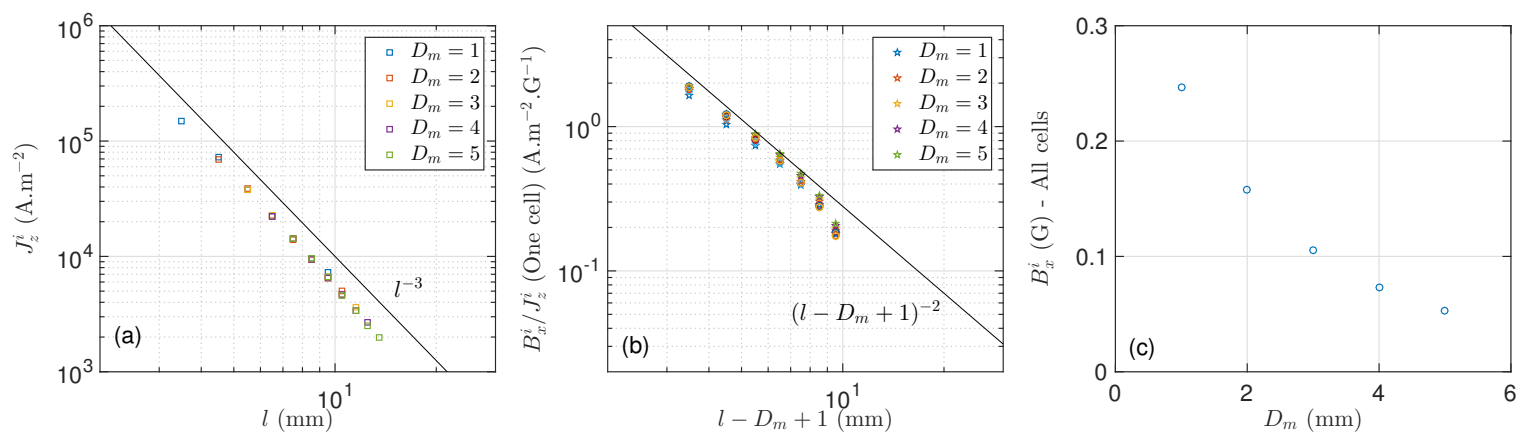

Figure 6. (a) Vertical induced current density in each cell (at $z=0$ ) as a function of $l$, distance from the cell to the center of the magnet, (b) induced magnetic field from each cell normalised by the vertical induced current density as a function of $l-D_{m}+1$, (c) $B_{x}^{i}$ induced from the whole pipe flow as a function of $D_{m}$.

We note that the induced current density at the center of the pipe scales as $l^{-3}$ and is thus proportional to the amplitude of the applied magnetic field. The amplitude of the induced magnetic field at the location of the sensor, from each of the individual cell, may then be approximated from Biot-Savart law, scaling as the inverse of the square of the distance between the cell and the sensor, i.e. $\left(l-D_{m}+1\right)^{-2}$. This is evidenced in Fig. 6(b). Note that, as $D_{m}$ increases, the volume probed by the magnetic distortion probe increases, but the absolute amplitude of the signal decreases, as seen in Fig. 6(c), which could limit the accuracy of the measurement.

A representation of the streamwise spatial resolution is less straightforward; however the extent of the flow probed in the streamwise direction may be estimated from the analysis of the vertical induced current density $J_{i}^{z}$ in the $(x, z)$ plane at $y=3 \mathrm{~mm}$. Fig. 7 displays the intensity of the applied magnetic field (panels (a) and (c)) and the normalised vertical induced current density (panels (b) and (d)) for the two extreme values of $D_{m}$, namely $1 \mathrm{~mm}$ (panels (a) and (b)) and $5 \mathrm{~mm}$ (panels (c) and (d)). A first 


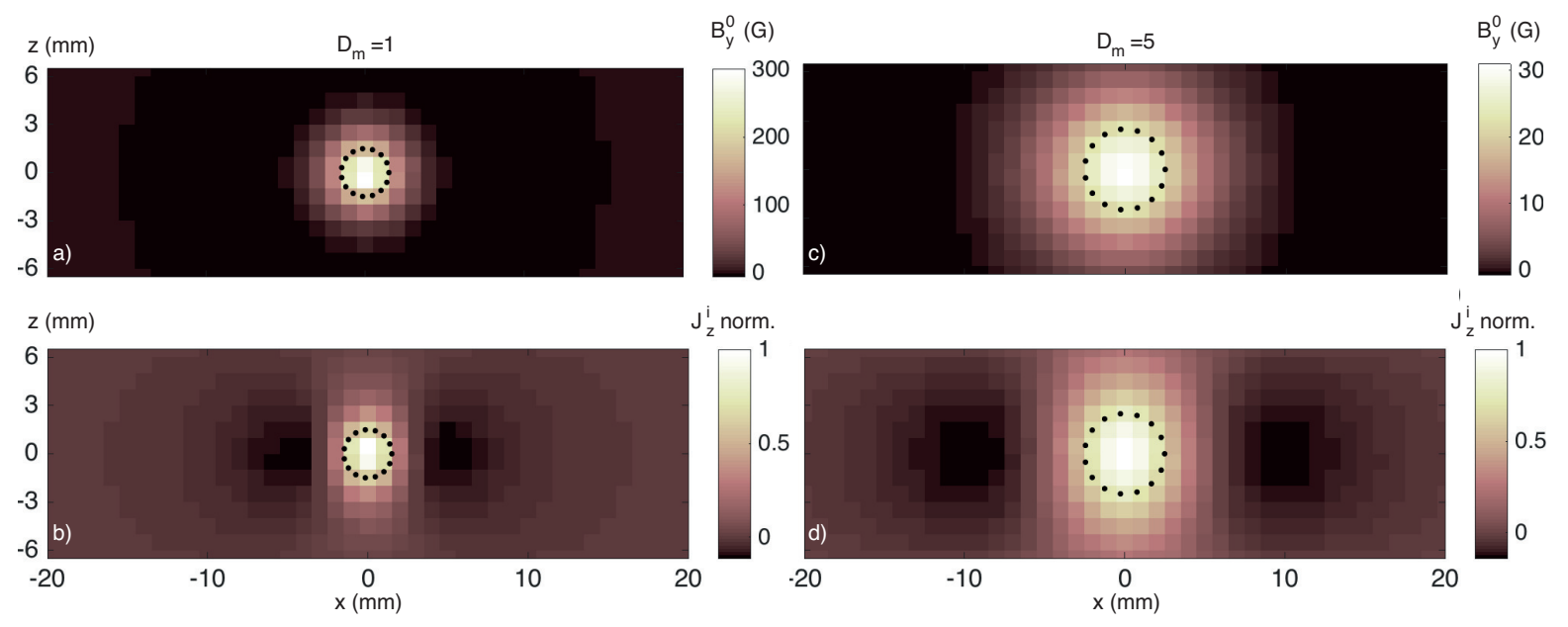

Figure 7. (a) and (c) Intensity of the applied magnetic field and (b) and (d) normalised vertical induced current density profiles in the $(x, z)$ plane at $y=3 \mathrm{~mm}$ for (a) and (b) $D_{m}=1 \mathrm{~mm}$ and (c) and (d) $D_{m}=5 \mathrm{~mm}$.

observation is that the profile of the intensity of the vertical induced current (driven by the electromotive force $(u \mathbf{x}) \times\left(B^{0} \mathbf{y}\right)$ since the applied magnetic field is mainly in the $y$-direction) is very similar to the intensity of the applied magnetic field. To gain further insight, the circles displayed in Fig. 7(a) and (c) as black dots show a region where the intensity of the magnetic field is $70 \%$ of the maximum in the plane. These circles are reproduced in Fig. $7(\mathrm{~b})$ and (d) and match the region in which the induced current density is larger that $68 \%$ of the maximum. The conclusions drawn previously about the spanwise extent is thus valid in the streamwise direction: the extent of the flow probed by the magnetic distortion probe is set by the homogeneity of the applied magnetic field and may be computed from the magnetic field profile.

As a partial conclusion, several features at leading order emerge from our numerical model in the simple configuration with a single magnet in the vicinity of the flow:

- The induced current in the flow is proportional to the intensity of the applied magnetic field.

- The extent of the probed flow is given by the extent of homogeneous amplitude of the applied magnetic field.

- The induced magnetic field at a given location is fairly well understood from the Biot-Savart law taking into account the induced current.

From these features, the basics for optimal configurations allowing for flowrate measurements are the following: (i) the most homogeneous profile of applied magnetic field throughout the pipe cross section, (ii) magnetic field sensors located at positions chosen such as to compensate for the inhomogeneity of the applied magnetic field (i.e. the sensing location has to be closer to pipe areas where the amplitude of the applied magnetic field are the lowest. It thus appear that, in the Hallbach array presented in sections 2 and 3, the optimal configuration requires to select an Hallbach array 
that imposes the most homogeneous $B^{0}$ within the cross-section and sensors located midway between the $y=0$ plane and the magnet face to compensate for the spatial inhomogeneity of the induced current density. We emphasize that while the above mentioned features allow to identify the behavior of the magnetic distortion probe at leading order, the detailed analysis of the spatial resolution also depends on the way induced currents loop back within the flow or the pipe, and thus depends on the exact geometry and electromagnetic boundary conditions.

\section{Conclusion}

In this article, an accurate numerical model of the contactless magnetic distortion probe has been introduced. This numerical model accurately reproduces the stationary regimes observed in a dedicated experimental pipe flow benchmark for several flow and applied magnetic field configurations. The calibration procedure of the magnetic distortion probe, as for all of the electromagnetic flowmeter or velocimeter, usually require extensive experimental tests. In the context of the magnetic distortion probe, this task may now be simplified thanks to the use of our numerical model.

Moreover, our numerical model is a valuable tool used to investigate the spatial extent of the flow probed by the magnetic distortion probe. In particular, we discussed the features leading to local velocity measurements or global flowrate measurements. The optimal arrangement required to probe global flowrates in pipes was finally discussed based on the numerical model.

\section{Acknowledgements}

The authors thank D. Cébron for his valuable time and numerous advices when starting using COMSOL. The authors acknowledge financial support from PULSALYS under contract L700.

\section{References}

[1] T Schulenberg and R Stieglitz. Flow measurement techniques in heavy liquid metals. Nucl. Eng. Des., 240,2077-2087, (2010).

[2] S Eckert, A Cramer, and G Gerbeth. Velocity measurement techniques for liquid metal flows. Magnetohydrodynamics: Historical Evolution and Trends, Fluid Mech. A., 80, 275-294, (2007).

[3] Eckert S, Buchenau D, Gerbeth G, Stefani F and Weiss FP Some recent developments in the field of measuring techniques and instrumentation for liquid metal flows J. Nucl. Sci. Techn., 48 490-499, (2011)

[4] Y Takeda Measurement of velocity profile of mercury flow by ultrasound Doppler-shift method. Nucl. Technol., 79, 120-124, (1987).

[5] D Brito, HC Nataf, P Cardin, J Aubert, and JP Masson. Ultrasonic Doppler velocimetry in liquid gallium. Exp. Fluids, 31, 653-663, (2001).

[6] Y Saito, K Mishima, Y Tobita, T Suzuki, and M Matsubayashi. Measurements of liquidmetal two-phase flow by using neutron radiography and electrical conductivity probe. Exp. Therm. Fluid Sci., 29,323-330, (2005). 
[7] Lehde H, Lang WT. Device for measuring rate of fluid flow US Patent 2435043 (1948)

[8] A Pavlinov, R Khalilov, A Mamikyn and I Kolesnichenko. Eddy current flowmeter for sodium flow. J. Phys. Conf. Ser., 208, 012031, (2017).

[9] Priede J, Buchenau D and Gerbeth G. Contactless electromagnetic phase-shift flowmeter for liquid metals Meas. Sci. Techn. 22055402 (2011)

[10] Stefani F and Gerbeth G. A contactless method for velocity reconstruction in electrically conducting fluids Meas. Sci. Techn., 11 758-765 (2000)

[11] M Ratajczak, $\mathrm{T}$ Gundrum, $\mathrm{F}$ Stefani, and $\mathrm{T}$ Wondrak Contactless Inductive Flow Tomography: Brief History and Recent Developments in Its Application to Continuous Casting Journal of Sensors, 2014, 739161, (2014).

[12] A Thess, EV Votyakov, and Y Kolesnikov. Lorentz force velocimetry. Phys. Rev. Lett., 96,164501, (2006).

[13] C Heinicke Spatially resolved measurements in a liquid metal flow with Lorentz force velocimetry. Exp. Fluids, 54,1560, (2013).

[14] Miralles S, Verhille G, Plihon N, Pinton JF. The magnetic-distortion probe: velocimetry in conducting fluids, Rev. Sci. Instrum., 82095112 (2011)

[15] Pinton JF, Plihon N, Verhille G, Miralles S. Velocimetre and method for measuring the speed of flow of an electrically conducting fluid, Patent WO2011151257 (2011)

[16] J. Mallinson. One-sided fluxes - a magnetic curiosity?, IEEE Trans. Magn. 9, 678-682 (1973)

[17] Michael Werner and Bernd Halbedel. IEEE Trans. Magn. 48, 2925-2928 (2012)

[18] D. Cébron, M. Le Bars, P. Maubert, P. Le Gal, Magnetohydrodynamic simulations of the elliptical instability in triaxial ellipsoids Geophys. Astrophys. Fluid Dyn., 106, 524-546 (2012)

[19] Z. Stelzer, D. Cébron, S. Miralles, J. Noir, S. Vantieghem and A. Jackson, Experimental and numerical study of electrically-driven MHD flow in a modified cylindrical annulus: (2) Instabilities, Physi. Fluids 27, 084108 (2015).

[20] A. Jackson, A. Sheyko, P. Marti, A. Tilgner, D. Cébron, S. Vantieghem, R. Simitev, F.H. Busse, X. Zhan, G. Schubert, S. Takehiro, Y. Sasaki, Y. Hayashi, A. Ribeiro, C. Nore, J-L Guermond, A spherical shell numerical dynamo benchmark with pseudo vacuum magnetic boundary conditions Geophys. J. Int., 196 (2) : 712-723 (2014) 